\title{
Analysis of Search Schemes in Cognitive Radio
}

\author{
Ling Luo and Sumit Roy \\ Department of Electrical Engineering \\ University of Washington, Seattle, WA 98195 \\ Email: \{luol,sroy\}@u.washington.edu
}

\begin{abstract}
Cognitive Radio systems face an important challenge - fast and reliable channel searching to enable secondary users to optimize available spectral resources. We revisit conventional urn models for channel occupancy and analyze the performance of several search schemes in terms of the mean time to detection. In particular, we focus on correlated Markov models for bin occupancy and highlight the performance of $n$-step serial search (nSS) algorithm
\end{abstract}

Index Terms - Cognitive Radio, channel model, search scheme

\section{INTRODUCTION}

Due to the rapidly increasing demands on wireless bandwidth, available spectral resources for new services are scarce, especially for $1-10 \mathrm{GHz}$ band. Recent measurements from FCC demonstrate that only $15-85 \%$ of this spectrum is utilized on (time) average [1]. These motivate Cognitive Radio (CR) [2,3] operation whereby secondary users are allowed to actively search and acquire idle channels (unused by primary users) provided they do not interfere with primary users' rights', i.e. use the spectrum on a non-interfering basis.

Fast and reliable channel searching is thus an important component for any CR network. Research on spectrum sensing has paid considerable attention to novel approaches based on cooperative sensing, dynamic spectrum access and spectral agility $[5,6,7,8,11]$. Different sensing strategies are apropos to emerging new CR network architectures, leading to the desire for a uniform metric for comparing different search strategies. We use the mean detection time drawing on random urn occupancy models [9] in this work. We seek to highlight the impact of different a) channel availability (occupancy) models and b) search strategies on the mean detection time for spectral scanning by secondary users; in particular, we investigate the differences between independent and Markov (correlated) occupancy models.

The rest of this paper is organized as follows. Detailed descriptions of channel models and search schemes are provided in Sections 2 and 3, respectively. We present the analysis of mean-time to detection in Section 4. Simulation results in Section 5 for each search strategy support the analysis and explore trade-offs among average detection time, power consumption and achievable probabilities of detection and false alarm. We conclude the paper in Section 6.

\section{Channel Models Description}

Each secondary user of a CR network attempts to sense the spectral band composed of a $N$-set of contiguous discrete frequency domain channels. At a given instant, the average number of idle channels is $L$ (both $N$ and $L$ are large, and $L / N<<1$ ). We assume that the detection probability $\left(P_{d}\right)$ and false alarm probability $\left(P_{f a}\right)$ pertinent to the sensing of any channel is the same. In the event of a false alarm, we assume that the sensing process requires $J$ sensing durations to recover and resume scanning, i.e. is the associated penalty.

\section{A. Identical Independent Distribution (i.i.d) Model}

In an i.i.d model, each discrete channel has the same probability $L / N$ to be idle. We define the binary variable $O_{k}$ to denote the status of the $k$ th channel $(k=1, \ldots \mathrm{N}$ denotes the $N$ channels in sequence) where $0(1)$ means that channel is busy (free).

$$
P\left(O_{k}=1\right)=\frac{L}{N}, k=1,2,3 \ldots N
$$

The traditional i.i.d models are considered as Random Occupancy. As shown in Fig.1, the $L$ randomly available (i.i.d) channels are scattered over the N-set. 


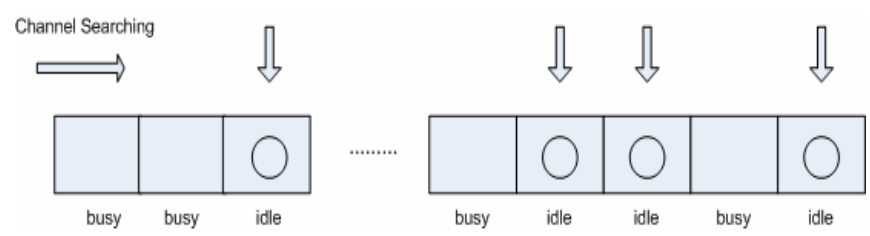

Fig.1 Random occupancy

\section{B. Correlated Markov Model}

To model channel clustering, we introduce Correlated Occupancy whereby the state $O_{k}$ for the $k$-th channel depends on those of it's neighbors. Using a first-order Markov dependence between two contiguous channels, we introduce the conditional probabilities: ( $\rho$ is transition probability)

$$
\begin{aligned}
& P\left(O_{k}=1 \mid O_{k+1}=1\right)=\rho \\
& P\left(O_{k}=0 \mid O_{k+1}=0\right)=\rho
\end{aligned}
$$

where the mean persistence time in each state is assumed identical. The model is described via a Markov Chain in Fig.2, with a transition matrix: (eigenvalues are 1 and $2 \rho-1)$

$$
\begin{aligned}
& \underline{P}^{\prime}=\left(\begin{array}{cc}
\rho & 1-\rho \\
1-\rho & \rho
\end{array}\right) \\
& \underline{P}^{\prime}=\sum_{k=0}^{N-1} \underline{P}^{(k)}=\left(\begin{array}{ccc}
\frac{N}{2}+\frac{1-(2 \rho-1)^{N}}{4(1-\rho)} & \frac{N}{2}-\frac{1-(2 \rho-1)^{N}}{4(1-\rho)} \\
\frac{N}{2}-\frac{1-(2 \rho-1)^{N}}{4(1-\rho)} & \frac{N}{2}+\frac{1-(2 \rho-1)^{N}}{4(1-\rho)}
\end{array}\right)
\end{aligned}
$$

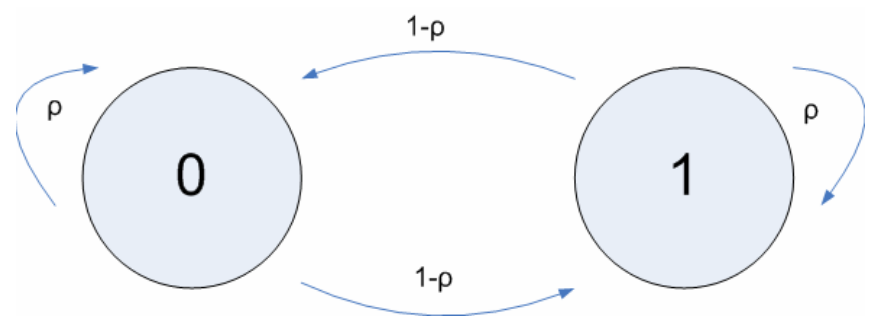

Fig.2 Correlated occupancy

We let the expected number of idle channels in this case equal $L$ which is the sum of the probabilities that each channel is idle. Assuming that the Markov Chain (I) starts from the status 0 , it follows using (II) $(N>2 L)$ :

$$
\begin{aligned}
& L=\sum_{k=1}^{N} P\left(O_{k}=1\right)=\underline{P^{\prime}}[0,1] \\
& =\frac{N}{2}-\frac{1-(2 \rho-1)^{N}}{4(1-\rho)} \approx \frac{N}{2}-\frac{1}{4(1-\rho)} \\
& \rho=1-\frac{1}{2(N-2 L)}
\end{aligned}
$$

\section{SEARCH SCHEMES}

\section{A. Random Search}

Random search is a basic search strategy in CR: the secondary user randomly picks up a channel to detect whether it is idle or not. If it is detected to be busy, the user then resorts to searching another channel randomly. The search stops when an idle channel is found.

\section{B. Serial Search}

In serial search, the secondary user searches channels in sequence till an idle channel is discovered. The state diagram for serial search is described in Fig.3, where the secondary user chooses the initial channel randomly to start searching. If an idle channel is not detected in one pass, the search is again re-initialized.

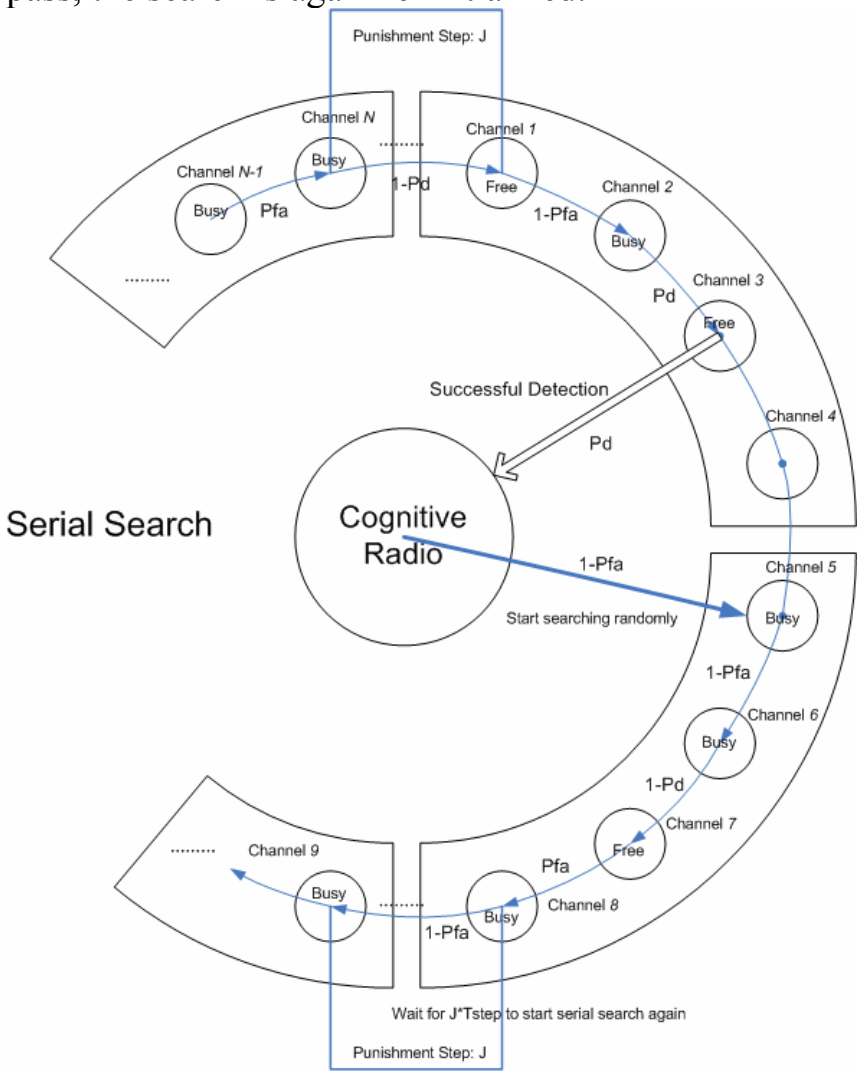

Fig.3 Serial search

\section{C. n-step Serial Search}

This is the generalization of the standard serial search with step size larger than 1. However, as shown in Fig.4, the step size $n<L$ to ensure that a cluster of free channels is not missed. 


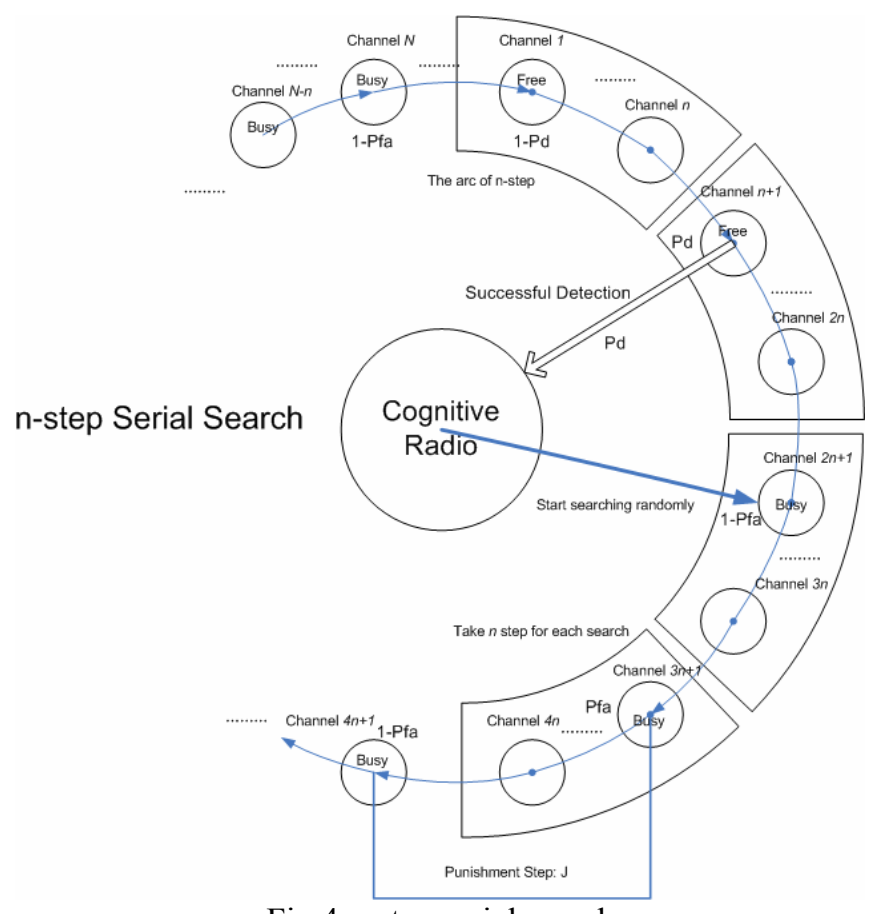

Fig.4 n-step serial search

\section{Analysis of AVERAge Detection Time}

Each channel scanning step has two components:

i. A fixed duration for the receiver to switch its sensing circuitry to the new channel; this is assumed to be a constant value $T_{c}$, regardless of magnitude of the jump in frequency domain at each scanning step, and largely depends on the circuit implementation.

ii. The duration $\mathrm{T}_{\text {sens }}$ for reaching a decision on channel status (busy/idle); this is a function solely of the desired $\mathrm{P}_{\mathrm{d}}-\mathrm{P}_{\mathrm{fa}}$ which are assumed constant for each channel.

Thus the average detection (acquisition) duration can be written as:

$$
\overline{T_{a c q}}=\overline{S_{a c q}} \times\left(T_{c}+T_{\text {sens }}\right)
$$

where $\overline{S_{a c q}}$ is the average number of steps in channel scanning prior to a success (i.e. detecting an idle channel). In our analysis below, we focus on the derivation of the average number of sensing steps needed prior to detection of an idle channel.

\section{A. Sensing Duration}

In a typical energy detection model, the received signal samples are filtered to a detector bandwidth $\left(\mathrm{B}_{\text {sense }}\right)$, passed through a square law detector, and integrated for a set duration $\left(\mathrm{T}_{\text {sense }}\right)$ before the accumulated energy is compared with a decision threshold $\left(D_{t}\right)$. Here $B_{\text {sense }}$ is the bandwidth of one discrete channel, which is set to $1 \mathrm{MHz}$.

From [13], the following equations can approximate $\mathrm{P}_{\mathrm{d}}$ and $\mathrm{P}_{\mathrm{fa}}$ of the detector:

$$
\begin{aligned}
P_{d} & \approx Q\left(\frac{D_{t}-2 T_{\text {sense }} B_{\text {sense }}(1+S N R)}{2 \sqrt{T_{\text {sense }} B_{\text {sense }}} \sqrt{1+2 S N R}}\right) \\
P_{f a} & \approx Q\left(\frac{D_{t}-2 T_{\text {sense }} B_{\text {sense }}}{2 \sqrt{T_{\text {sense }} B_{\text {sense }}}}\right)
\end{aligned}
$$

The function $\mathrm{Q}(\mathrm{x})$ is defined as:

$$
Q(x)=\frac{1}{\sqrt{2 \pi}} \int_{x}^{\infty} e^{-\tau^{2} / 2} d \tau
$$

In this paper, $\mathrm{SNR}\left(P_{\text {signal }} / P_{\text {noise }}\right)$ is set to $3 \mathrm{~dB} ; \mathrm{P}_{\mathrm{d}}$ is 0.9 and $\mathrm{P}_{\mathrm{fa}}$ is 0.05 . From (10)-(12), we can then obtain that:

$$
T_{\text {sense }} \approx 5.1 \times 10^{-3} \mathrm{~ms}<<1 \mathrm{~ms}
$$

Compared to the value of $\mathrm{T}_{\mathrm{c}}$, the sensing duration $\mathrm{T}_{\text {sense }}$ for each search step can be ignored in our work.

\section{B. Serial Search under Random Occupancy}

We define a set of random variables $\left\{X_{k}\right\}$ as the location of the $k$-th free channel in the available spectrum. For instance, if the 1 st idle channel is the 4 th channel, then $X_{1}=4$. In Random Occupancy, $X_{k} \sim U(0, N)$ and are mutually i.i.d.

$P_{d}=1, P_{f a}=0$ (Ideal): $\quad$ In this ideal scenario, the average number of detection steps is the mean distance from any starting point (nominally $\mathrm{k}=0$ ) to the nearest one of these $L$ random variables of $\left\{X_{k}\right\}$. Let $Y$ denote the steps required by serial search to reach an idle channel, then:

$Y=\min \left(X_{1}, X_{2} \ldots, X_{L}\right)$

Thus the distribution of $\{Y\}$ and the average steps are:

$1-F_{Y}(y)=\left(1-F_{X}(y)\right)^{L}$

$\overline{S_{a c q}}=\int_{0}^{N-L} y f_{Y}(y) d y=N L\left[\frac{1-(L / N)^{L}}{L}-\frac{1-(L / N)^{L+1}}{L+1}\right]$

For, $L$ and $N$ large:

$\overline{S_{a c q}} \approx N /(L+1)$

$P_{d}<1, P_{f a}>0$ (Non-ideal): We split the number of steps for serial search in this scenario into two parts: $S_{\text {acq }}=S_{\text {normal }}+S_{\text {punish }}$, where $S_{\text {normal }}$ and $S_{\text {punish }}$ respectively denote the number steps to detect an idle channel based on $P_{d}<1$ and the penalty due to non-zero false alarms in each search cycle. The probability that serial search successfully detects an idle channel is $P_{d} L / N$ for each attempt. Therefore, it can be treated as the case of i.i.d 
variables $\left\{X_{k}\right\}$ that are uniformly distributed in $(0$, $\left.N / P_{d}\right)$.

$\overline{S_{\text {normal }}}=\frac{N}{P_{d}} L\left[\frac{1-\left(P_{d} L / N\right)^{L}}{L}-\frac{1-\left(P_{d} L / N\right)^{L+1}}{L+1}\right]$

Let $y$ ' denote the number of steps in normal search and $u$ denote the times of occurrence of false alarm during each search cycle. It can be concluded that $u$ follows Binomial Distribution: $u \sim B\left(y^{\prime},(N-L) P_{f a} / N\right)$. The average numbers of false alarm and penalty steps are

$\overline{S_{f a}}=\sum_{u=0}^{\infty} u f(u)=\sum_{u=0}^{\infty} u \int_{0}^{N / P_{d}-L} f\left(u \mid S_{\text {normal }}=y^{\prime}\right) f_{Y}\left(y^{\prime}\right) d y$

$\overline{S_{\text {punish }}}=J \overline{S_{f a}}=\frac{N-L}{N} J P_{f a} \overline{S_{\text {normal }}}$

Therefore, from (17)-(19), the average number of steps for success of serial search under random occupancy is:

$\overline{S_{a c q}}=\frac{(N-L) J P_{f a}+N}{P_{d}} L\left[\frac{1-\left(P_{d} L / N\right)^{L}}{L}-\frac{1-\left(P_{d} L / N\right)^{L+1}}{L+1}\right]$

$\overline{S_{a c q}} \approx \frac{(N-L) J P_{f a}+N}{P_{d}(L+1)}$

\section{Random Search under i.i.d Models}

In i.i.d models, the probability of each attempt for a secondary user to randomly catch an idle channel is the same. Similar to the analysis in Part B of Section 4, we can conclude that the number of steps for random search follows a Geometric Distribution. Thereby its average value is given by:

$\overline{S_{a c q}}=\left(\frac{N-L}{N} J P_{f a}+1\right) \frac{N}{P_{d} L}=\frac{P_{f a} J(N-L)+N}{L P_{d}}$

\section{Random Search under Correlated Occupancy}

In correlated occupancy, if there are $m$ idle channels in the spectrum with probability $p_{m}$, then we can obtain the average number of steps to success from (21):

$E[$ Step $\mid$ idle_channel $: m]=\frac{P_{f a} J(N-m)+N}{m P_{d}}$

If the 1st channel is assumed to be busy, then the probability that all $N$ channels are unavailable

$P\left(Q=Q_{2}=\ldots=O_{N}=0\right)$

$=P(Q=0) P\left(O_{2}=0 \mid Q=0\right) . P\left(O_{N}=0 \mid O_{N-1}=. .=Q=0\right)=\rho^{N-1}$

Neither random nor serial Search can find an idle channel in this situation. During the time the Markov Chain transitions to the next state, the number of search step is constant and set equal to $N$. It can be observed from transition matrix (I) that:
$P_{00}=P_{11}=P_{\text {state-unchanged }}=\rho$

$P_{01}=P_{10}=P_{\text {state-changed }}=1-\rho$

To calculate the probability of $m$ idle channels, we consider the case that they occur as $k$ groups. There will thus be typically $2 k$ state transitions ${ }^{1}$. Thus the probability that $m$ idle channels appear in the Markov Chain (I) is:

$P(m-$ idle $) \approx \sum_{k=1}^{m} C_{m-1}^{k-1} C_{N-m}^{k} P_{\text {state-unchanged }}^{N-1-2 k} P_{\text {state-changed }}^{2 k}$

(26) is based on the assumption that the number of idle channels is less than busy channels $(m<N-m)$. In case of $m>N-m$, the probability of $m$ idle channels in the spectrum is given by:

$P(m-$ idle $) \approx \sum_{k=1}^{N-m-1} C_{N-m-2}^{k-1} C_{m+1}^{k} P_{\text {state-unchanged }}^{N-1-2 k} P_{\text {state-changed }}^{2 k}$

Therefore, from (23)-(27), the average number of steps is:

$\overline{S_{\text {acq }}}=E[E[$ Step $\mid m-$ idle $]]$

$=\rho^{N-1} N+\sum_{m=1}^{N-1} \sum_{k=1}^{\min (m)} C_{\min (m)-1}^{k-1} C_{\max (m)+1}^{k} \rho^{N-1-2 k}(1-\rho)^{2 k}$

$\min (m)=\min (m, N-m), \max (m)=\max (m, N-m)$

\section{E. Serial Search under Correlated Occupancy}

We define four search states: State 0 denotes the successful detection of an idle channel; State 1 the unsuccessful detection of an idle channel; State 2 the successful detection of a busy channel; State 3 the false alarm of a busy channel. In any search algorithm, State 0 is an absorbing state, which means that the search is ended once an idle channel is successfully detected. The transition diagram for this chain is shown in Fig.5, where the transition matrix $\underline{P}$ is:

$\underline{P}=\left(\begin{array}{cccc}1 & 0 & 0 & 0 \\ \rho P_{d} & \rho\left(1-P_{d}\right) & (1-\rho)\left(1-P_{f a}\right) & (1-\rho) P_{f a} \\ (1-\rho) P_{d} & (1-\rho)\left(1-P_{d}\right) & \rho\left(1-P_{f a}\right) & \rho P_{f a} \\ (1-\rho) P_{d} & (1-\rho)\left(1-P_{d}\right) & \rho\left(1-P_{f a}\right) & \rho P_{f a}\end{array}\right)$

\footnotetext{
${ }^{1}$ If a group occurs at the beginning or end, the number of transitions equals $2 \mathrm{k}-1$. We ignore this boundary condition, for $\mathrm{N}, \mathrm{k}$ large.
} 


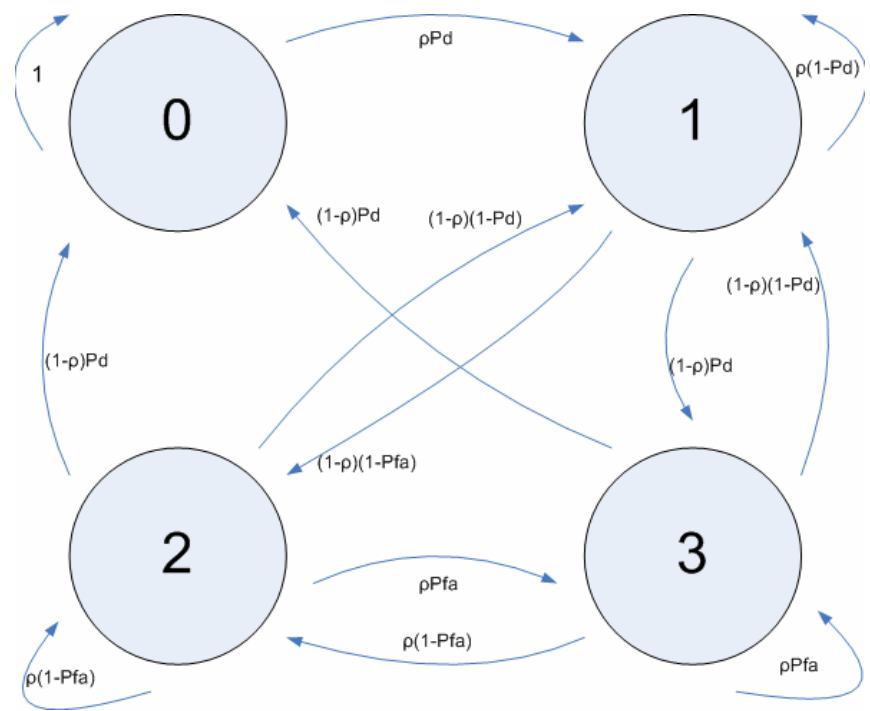

Fig.5 Transition diagram for search states

Since the number of idle channels is $L$ out of a total of $N$, it follows that:

$$
\begin{aligned}
& P\{S=0\}=\frac{L}{N} P_{d}, P\{S=1\}=\frac{L}{N}\left(1-P_{d}\right) \\
& P\{S=2\}=\frac{N-L}{N}\left(1-P_{f a}\right), P\{S=3\}=\frac{N-L}{N} P_{f a}
\end{aligned}
$$

If there is no penalty due to a false alarm $(\mathrm{J}=0)$ the average number of steps to reach the idle channel is:

$\overline{S_{\text {acq }}}=\sum_{k=1}^{\infty} k P\left\{S_{\text {acq }}(k)\right\}=\sum_{k=1}^{\infty} P\left\{S_{\text {acq }} \geq k\right\}$

For $S_{\text {acq }} \geq k$, it is necessary that in the first $k$ steps the Markov Chain (III) has not entered the absorbing State 0 [12]. Since the first step of serial search might start from State $i$ : $(i=1,2,3)$ :

$$
\begin{aligned}
& P\left\{S_{\text {acq }} \geq k\right\}=\sum_{i=1}^{3} P\left\{S_{\text {acq }} \geq k \mid S=i\right\} P\{S=i\} \\
& =\sum_{i=1}^{3}\left[P\{S=i\}\left(\underline{P}_{i, 1}^{(k-1)}+\underline{P}_{i .2}^{(k-1)}+\underline{P}_{i, 3}^{(k-1)}\right)\right]
\end{aligned}
$$

As a result of the property of absorbing state, we identify the following sub- matrix $\underline{P}$, denoted as $\underline{A}$ below.

$$
\underline{A}=\left(\begin{array}{ccc}
\rho\left(1-P_{d}\right) & (1-\rho)\left(1-P_{f a}\right) & (1-\rho) P_{f a} \\
(1-\rho)\left(1-P_{d}\right) & \rho\left(1-P_{f a}\right) & \rho P_{f a} \\
(1-\rho)\left(1-P_{d}\right) & \rho\left(1-P_{f a}\right) & \rho P_{f a}
\end{array}\right)
$$

Thereby the formula (31) can be transformed into

$$
P\left\{S_{a c q} \geq k\right\}=\sum_{i=1}^{2}\left(\underline{A}_{i, 1}^{(k-1)}+\underline{A}_{i .2}^{(k-1)}+\underline{A}_{i, 3}^{(k-1)}\right)
$$

Assuming that the matrix power series converges, the average number of detection steps without any false alarm penalty can be written as:

$$
\begin{aligned}
& \underline{N}=\sum_{k=0}^{\infty} \underline{A}^{(k)}=(I-\underline{A})^{-1} \\
& \left.\overline{S_{a c q}}=\sum_{k=1}^{\infty} P\left\{S_{a c q} \geq k\right\}=\sum_{i=1}^{3} P\{S=i\}\left(\underline{N}_{i, 1}+\underline{N}_{i .2}+\underline{N}_{i, 3}\right)\right]
\end{aligned}
$$

Meanwhile, the matrix $\underline{N}$ can be obtained by (33), and the specific calculation can be easily run by some computer software. We next incorporate the penalty due to false alarm by letting the secondary user wait for another $J$ steps to start the search algorithm again in the event of a false alarm of an idle channel. In such a case, the number of steps in State 3 will be multiplied by $J$. Therefore, the mean number of detection steps in the general case is given by

$$
\overline{S_{a c q}}=\sum_{i=1}^{3}\left[P\{S=i\}\left(\underline{N}_{i, 1}+\underline{N}_{i .2}+J \underline{N}_{i, 3}\right)\right]
$$

\section{F. n-step Serial Search under Correlated Occupancy}

In $n$-step serial search, the transition matrix $\underline{P^{\prime}}$ is given by $\underline{P}^{(n)}$, the n-step transition matrix of the original Markov Chain. Thus the average number of detection steps

$$
\begin{aligned}
& \overline{S_{a c q}}=\sum_{k=1}^{\infty} k P\left\{S_{a c q}(k)\right\}=\sum_{k=1}^{\infty} P\left\{S_{a c q} \geq k\right\} \\
& =\sum_{k=1}^{\infty} \sum_{i=1}^{3}\left[P\{S=i\}\left(\underline{P}_{i, 1}^{\prime(k-1)}+\underline{P}_{i .2}^{(k-1)}+\underline{P}_{i, 3}^{\prime(k-1)}\right)\right]
\end{aligned}
$$

Assuming convergence of the matrix power series, we find the average number of detection steps

$$
\overline{S_{\text {acq }}}=\sum_{i=1}^{3}\left[P\{S=i\}\left(\underline{N}_{i, 1}^{\prime}+\underline{N}_{i .2}^{\prime}+J \underline{N}_{i, 3}^{\prime}\right)\right]
$$

where $\underline{N}^{\prime}=\sum_{k=0}^{\infty} \underline{A}^{(k)}=\left(I-\underline{A}^{(n)}\right)^{-1}$

\section{Simulation Results}

\section{A. Experimental Environment}

We simulated the three search schemes mentioned above to evaluate their performance. In correlated occupancy, the transition matrix parameter $\rho$ is based on (8). The basic parameters of the simulation environment are: $N=50, P_{d}=0.9, P_{f a}=0.05, J=4$. Matlab is used to generate discrete channels, and the data are gathered by running 3000 realizations for each experiment.

\section{B. Average Number of Detection Step}

The number of idle channels is altered to investigate the performance of each search algorithm, shown in Fig.6. We can observe that serial search requires less 
detection time than random search under random occupancy. From (8), it can be concluded that as the number of idle channels is decreased, the transition probability increases to 1 , in correlated occupancy. When the transition probability is larger than 0.97 (corresponding to the case that there are no more than 16 idle channels), the free and busy channels have a high probability of clustering. When the transition probability is below 0.85 (the proportion of idle channel is higher than 0.47) for correlated occupancy, the difference in the mean number of steps required between random and serial search is small, as expected.

It can be also observed that the average number of steps sharply decreases as the number of idle channels goes up (transition probability rises) in correlated occupancy. The larger values of transition probability correspond to higher probability of consecutive busy channels and significant increase in the average number of detection steps. With the increase in the transition probability, the average length of a cluster of consecutive idle channels increases, implying reduction in $\mathrm{k}$. This in turn means that the approximations in (26) and (27) are less accurate and deviations among analysis and simulation results for both search schemes begin to show up in such scenario.

\section{Performance of n-step Serial Search}

The performance of $n$-step serial search under correlated occupancy is shown in Fig.7. Of particular interest is the cross-over of the mean detection time for random search vs $n$-step serial search. Consider $n=$ 2-step serial search; random search performs better when the transition probability is less than 0.94 , while 2-step serial search dominates at the higher transition probability. Further, the mean detection time of n-step serial search decreases with increasing $n$.

\section{Channel Sensing ( $\left.T_{\text {sense }}\right)$}

Channel sensing is commonly performed using non-coherent energy detection; for a higher $\mathrm{P}_{\mathrm{d}}$ and lower $\mathrm{P}_{\mathrm{fa}}$, a receiver has to integrate the signal over a longer duration.

For $B_{\text {sense }}=1 \mathrm{MHz}, \quad S N R=3 \mathrm{~dB}, \quad$ the relationship between $T_{\text {sense }}$ and varying $\mathrm{P}_{\mathrm{d}}-\mathrm{P}_{\mathrm{fa}}$ is shown in Fig. 8 and
Fig.9. We can observe that the value of $\mathrm{T}_{\text {sense }}$ is typically much smaller than $T_{c}$ (switching duration) of $1 \mathrm{~ms}$, and was therefore neglected in our computations. However, as $T_{c}$ or $B_{\text {sense }}$ (sensing bandwidth) becomes smaller, $\mathrm{T}_{\text {sense }}$ will become a significant portion of the whole detection time. The impact of such variations will be explored in future.

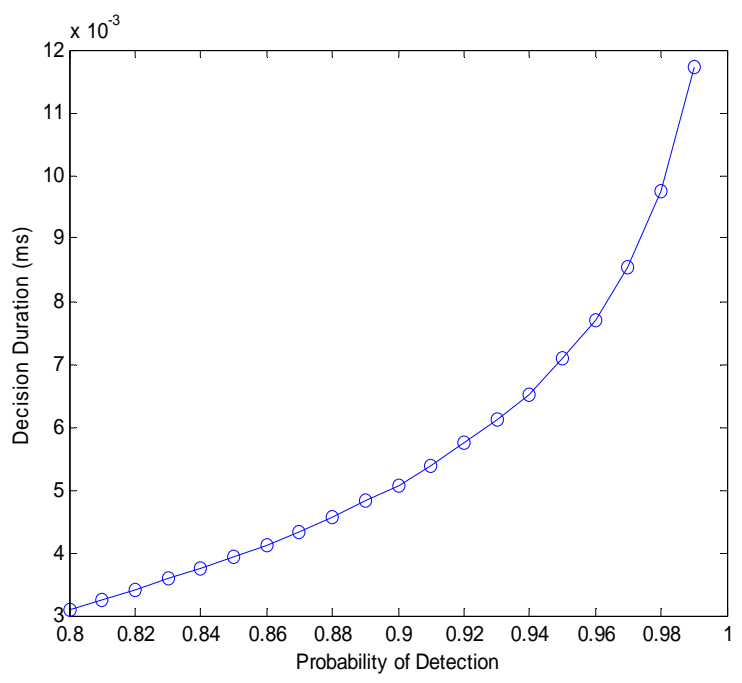

Fig.8 $T_{\text {sense }}$ for different $P_{d}$

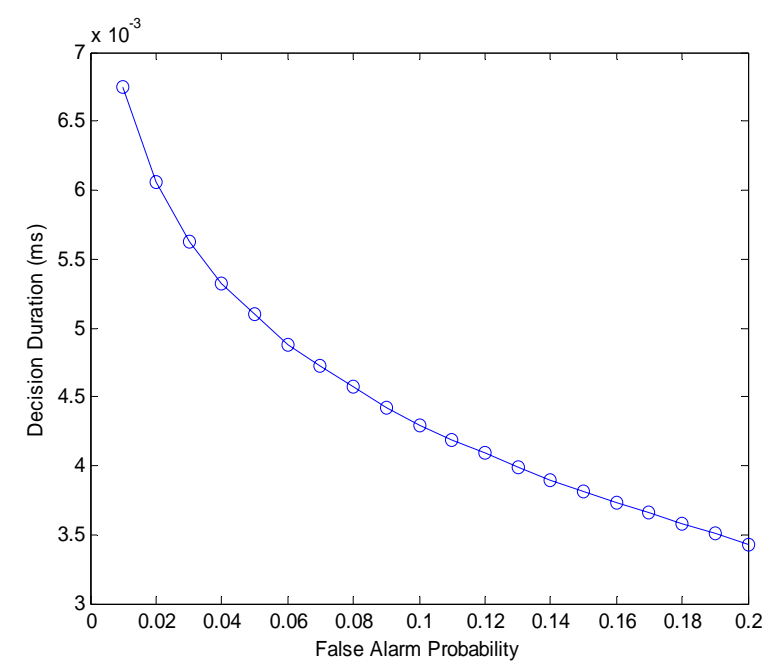

Fig.9 $\mathrm{T}_{\text {sense }}$ for different $\mathrm{P}_{\text {fa }}$ 


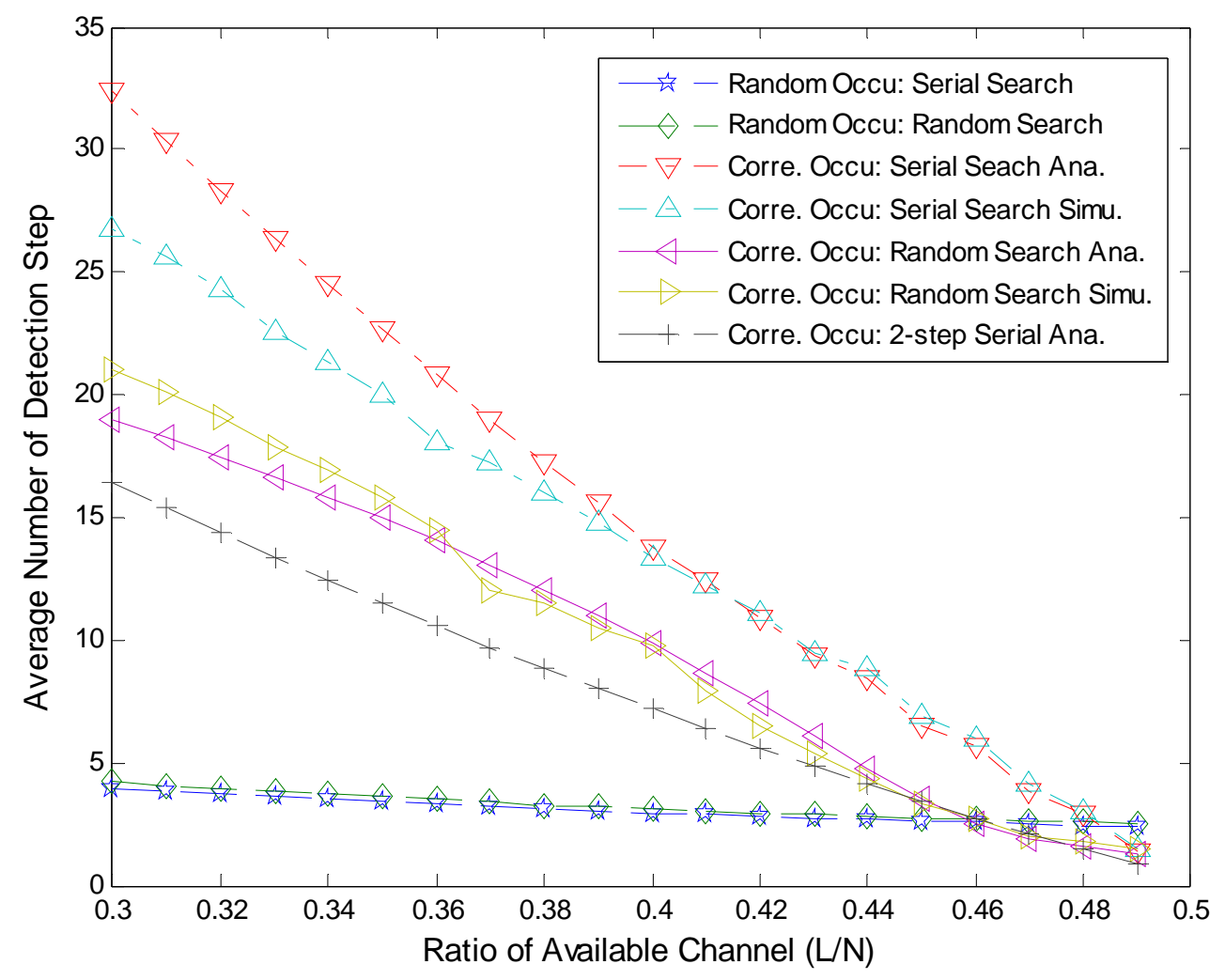

Fig.6 Average number of step for serial and random search

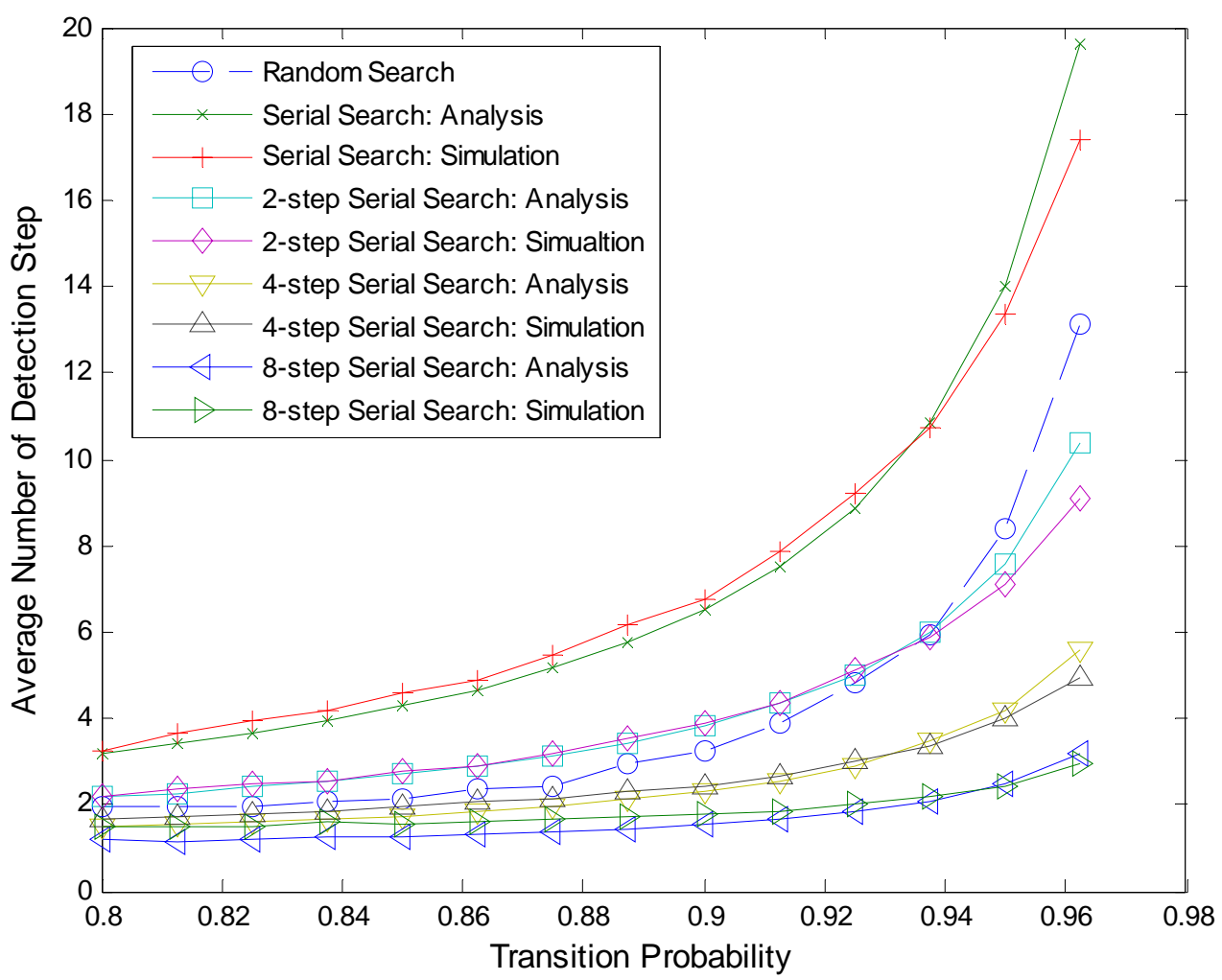

Fig.7 Performance of $n$-step serial search under correlated occupancy 


\section{CONCLUSION}

In this paper, we investigated the performance of random and serial search schemes for independent and correlated occupancy channel models. It is shown via analysis and simulation results that $n$-step serial search has lower detection time than random search. Furthermore, with the increase in the transition probability, performance in correlated occupancy channels to have the similar performance as clustering. The search schemes with less detecting time also have the high cost of energy, and our future work is to achieve a better equilibrium among these characters of CR network.

\section{REFERENCES}

[1] FCC, Spectrum Policy Task Force Report, ET Docket No.02-155, Nov. 2002

[2] J. Mitola and G.Q. Maguire, "Cognitive Radio: Making Software Radios More Personal", IEEE Personal Communications, Vol.6, Issue 4, pp.13-18, Aug. 1999

[3] S. Haykin, "Cognitive Radio: Brain-Empowered Wireless Communications", IEEE J. Select. Areas Commun., Vol.23, Issue 2, pp.201-220, Feb. 2005

[4] I.F. Akyildiz et al., "NeXt generation/dynamic spectrum access/cognitive radio wireless networks: A survey", Computer Networks, Vol.50, Issue 13, pp.2127-2159, Sep. 2006

[5] S.M. Mishra et al., "Cooperative Sensing among Cognitive Radio", IEEE ICC 2006, Vol.4, pp. 1658-1663, June 2006

[6] Y. Xing et al., "Dynamic Spectrum Access in Open Spectrum Wireless Networks", IEEE J. Select. Areas Commun., Vol.24, Issue 3, pp.626-637, Mar. 2006

[7] C.T. Chou et al., "What and How Much to Gain by Spectral Agility", submitted to IEEE/ACM Trans. On Networking, June 2004

[8] S. Mangold et al., "Spectrum Agile Radio: Radio Resource Measurements for Opportunistic Spectrum Usage", IEEE Globecom 2004, Vol.6, pp. 3467-3471, Nov. 2004

[9] O. Milenkovic and K.J. Compton, "Probabilistic Transforms for Combinatorial Urn Models", Combinatorics, Probability and Computing, Vol.13, Issue 4-5, pp.645-675, Jul. 2004

[10] A. Saleh and R. Valenzuela, "A Statistical Model for Indoor Multipath Propagation", IEEE J. Select. Areas Commun., Vol.5, Issue 2, pp.128-137, Feb. 1987
[11] R. Iyappan and S. Roy, "On Acquisition of Wideband Direct-Sequence Spread Spectrum Signals", IEEE Trans. On Wireless Commun., Vol.5, Issue 6, pp.1537-1546, June 2006

[12] S. Ross, Introduction to Probability Models, Academic Press, $8^{\text {th }}$ ED, 2003 